\title{
GEODA-GRUA: Adaptive Multibeam Conformal Antenna for Satellites Communications
}

\author{
J. García-Gasco Trujillo, Miguel Salas Natera, Ignacio Montesinos, Marta Arias Campo, M. Sierra Pérez and \\ Ramón Martínez
}

\begin{abstract}
Grupo de Radiación, Departamento de Señales, Sistemas y Radiocomunicación. Universidad Politécnica de Madrid. Avda. Complutense 30, 28040, Madrid, Spain. +34 915495700

\{jtrujillo; msalasn; nacho; marias; m.sierra.perez; ramon \}@gr.ssr.upm.es
\end{abstract}

\begin{abstract}
The GEODA-GRUA is one conformal adaptive antenna array designed for satellite communications. Operating at $1.7 \mathrm{GHz}$ with circular polarization, it is possible to track and communicate with several satellites at once being able to receive signals in full azimuth and within the range of $5^{\circ}$ to broadside elevation thanks to its adaptive beam. The complex structure of the antenna array has 2700 radiating elements based on a set of 60 similar triangular arrays that are divided in 15 subarrays of 3 radiating elements. A control module governs each transmission/receiver (T/R) module associated to each cell in order to manage beam steering by shifting phases.
\end{abstract}

\section{Introduction}

Nowadays, earth stations have as a common feature the use of large reflector antenna for downloading data from satellites [1]. Large reflectors have impairments such as mechanical complexity, low flexibility and high cost. Thus, the feasibility of other antenna technologies must be evaluated, such as conformal adaptive antennas based on multiple planar active arrays [2]. In the scenery under study, the capability to track several satellites simultaneously, higher flexibility, lower production and maintenance cost, modularity and a more efficient use of the spectrum; are the most important advantage to boost up active antenna arrays over large dishes.

The antenna GEODA-GRUA is a smart and conformal array system for satellite communications. As it is shown in Fig.1, its structure consists of a hemispherical dome placed on a cylinder of 1.5 meters height. Both cylinder and dome are conformed by 30 triangular planar arrays (Panels). Each Panel consists of 15 sub-arrays with three elements (Cells). The radiating element consists of two stacked circular patches. The principal patch is fed in quadrature in two points separated $90^{\circ}$ in order to get circular polarization. The smaller and coupled patch is used in the aim of improving the bandwidth.

$\mathrm{T} / \mathrm{R}$ modules are associated to each cell in order to select phase shifts between radiating elements. An electronic control module sets the steering direction of a triangular array by governing these T/R modules. Finally, the desired beam shape will be obtained by using the digital adaptive system of the signal of each triangular array. Thanks to this electronic control module and the resourcefulness structure conformed by 2.700 radiating elements, it is possible to communicate with satellites located within a spherical cap of $5^{\circ}$ of elevation.
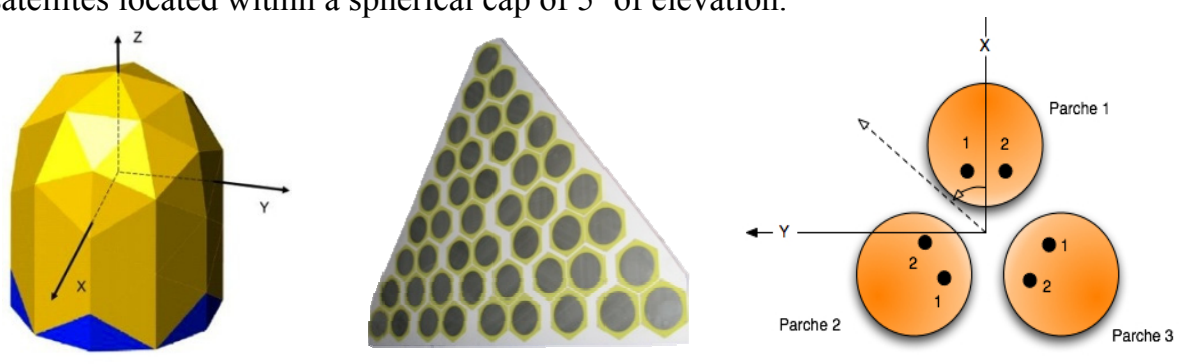

Fig 1. GEODA structure (left), panel (center) and cell (right)

At first, GEODA was thought as a reception antenna with single circular polarization. Reception module was designed, constructed and successfully measured. After GEODA good results and thinking about increasing the versatility of the antenna, GRUA project was born with the aim of providing to GEODA reception and transmission capability with double polarization. Hence, T/R module has also been designed, constructed and optimized, improving size and behavior. In this document will be presented the cell radiation pattern, the T/R module and control system. 


\section{Cell Radiation Pattern}

In the first place, the radiating pattern of the simple radiating element used to build the whole antenna was measured. As was presented in [3] and [4], lower than $1 \mathrm{~dB}$ of axial ratio level and good radiation pattern shape fulfilled specifications. Then, three elements array was manufactured. Since it has six ports (two ports per patch) and measurements were taken directly from the patch ports, they should be measured one by one and the global radiation pattern of the cell would be composed by means of mathematical calculation.

Anechoic chamber results are given in terms of $\mathrm{E} \theta \mathrm{y} \mathrm{E} \varphi$ of each port linear polarization. Next step is to simulate the phase difference that the hybrid coupler included between the ports of a double-stacked patch, so a $-90 \circ$ rotation angle was done in every secondary port. After that, it is mandatory to calculate the E $\theta$ y E $\varphi$ components of the entire cell. As the patches are rotated $120^{\circ}$ one from the contiguous, it is necessary to mathematically simulate the task of the phase shifters. Therefore, $120^{\circ}$ and $240 \circ$ should be taken into account.

Once the global radiated field is properly calculated, it is time to estimate the difference between copolar and crosspolar signals, and therefore, to calculate the axial ratio level for every sweeping angle.

Cell fulfills radiation requirements since it has a good polar - crosspolar level and a very low axial ratio. The radiation pattern shows symmetry and low side lobes for every $\varphi$ angle, as it can be seen in Fig.2 (left). Axial ratio level is under 1.5dB Fig.3 (right) for all the sweeping angles and reveals a flat pattern.
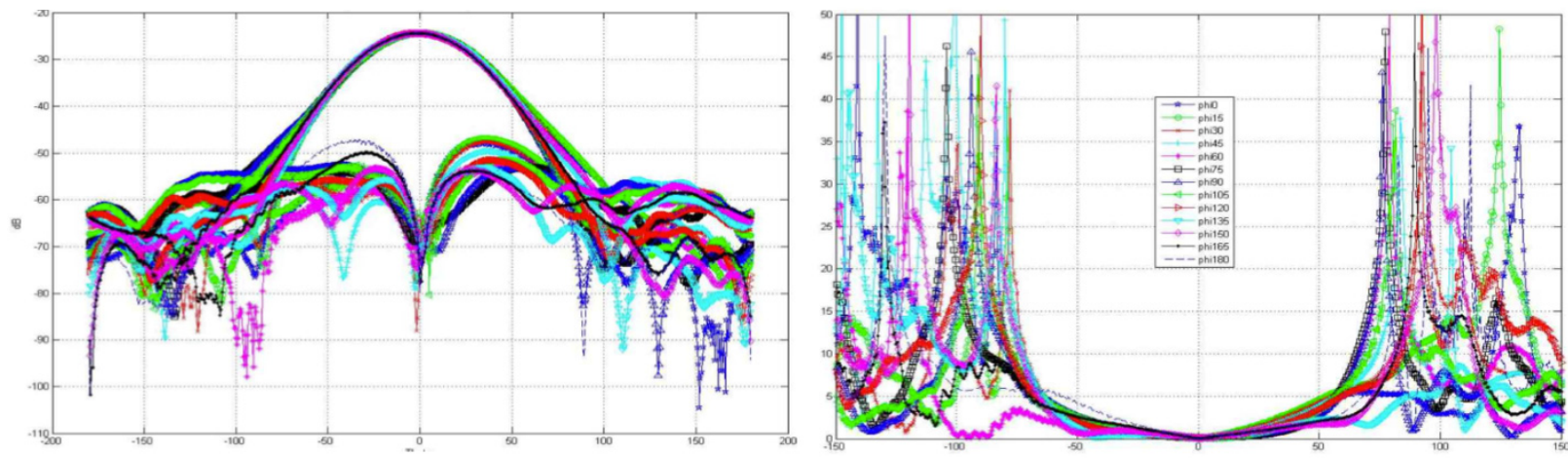

Fig 2. Cell radiation pattern (left). Cell radiation Capabilities (right)

Mutual coupling between feeding ports and between continuous patches and cells are very important aspects of the design and they have to be taken into account since it can modify the global panel radiation pattern. Regarding this, coupling level between patch ports is under $20 \mathrm{~dB}$ and between patches under $17 \mathrm{~dB}$ for broadside pointing direction. Further studies have been done in order to determine how the coupling affects to the array main beam pointing direction. They reveal that when the coupling effects increase, SLL becomes bigger and main beam becomes wider.

\section{T/R module}

Different T/R module configurations have been considered, providing either single or double polarization [5]. Fig. 3 shows the design implemented, which contain hybrid couplers, $2 \times 2$ switches, LNAs, phase shifters and $2 \times 1$ switches.

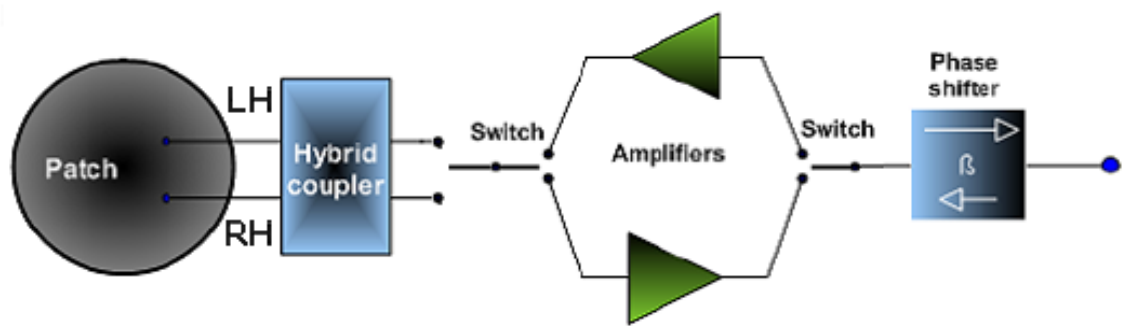

Fig 3. GEODA: Cell sub-array and RF circuit.

One goal of the use of this new T/R module is to optimize the space that each of them needs in every cell. Thus, some devices that had been designed using microstrip line technology in previous model has been replaced by microchips which work similarly but need much less space in the RF circuit. In addition, microstrip line configuration has been optimized, and smaller SMA components are used. 
In this module, considering reception way, the radiating element is connected to a coupler hybrid (QCN-25 Mini-Circuits) that will add circular polarization signals. Depending on the circular polarization received, left or right, the output will be in one of the hybrid coupler ports. Thus, a 2x2 ways switch (MASWSS0040 M/A-COM) leads the signal to the LNA (MCB13720 Freescale Semiconductor). Then, a 2x1 ways switch (PE4257 Peregrine Semiconductor) leads the signal to the $22.5^{\circ}$ step phase shifter (MAPS-008342-PKG003 M/A-COM 4 bits) to beamform the array. Thereby, the $2 \times 2$ ways switch needs 2 bits to control the polarization and $2 \times 1$ ways switch and LNA need 2 bits more to control the TX or RX operating mode. Hence, 8 bits are used to govern each T/R module. Fig. 4 shows T/R module test circuit.

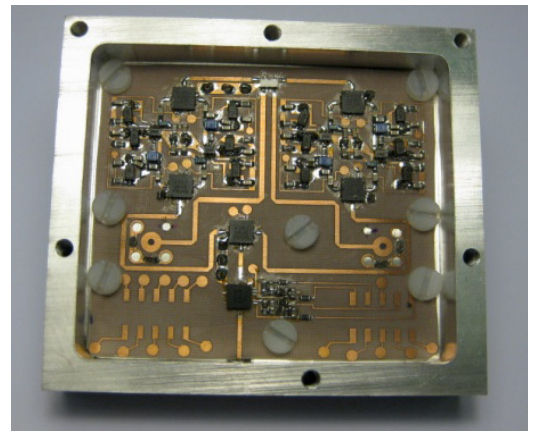

Fig 4. GEODA T/R module test circuit

The RF circuit of each Cell has three T/R modules combining the signal output. Since, it has reception and transmission duality, two switches $(2 \times 1)$ to select the RF path way are used with two bits of control each (same bits are used in the current design), and there are 2 bits more for test signal control. Thus, each Cell requires addressing 28 bits of control to state the system.

\section{GEODA Control System}

The control system has two main parts [6]: the hardware structure and the control software. A three-layers architecture has been implemented for the hardware structure (Fig 5). The first layer consist of one embedded microcontroller (PIC18F87J10) in each cell RF circuit to control the phase shifter, LNA and coupler switch for the test signal. The second layer has the panel microcontroller (PIC18F4550) that addresses the control data to each cell. The third layer consists on the PC (work station) that executes the control software. The PC is connected to the microcontroller board of the panel by an USB port, and this with the 15 microcontroller boards of each cell by I2C bus. The $1 \times 15$ I2C bus board was developed to isolate the $15 \mathrm{I} 2 \mathrm{C}$ bus ports controlling the capacitance level of the wire connections.

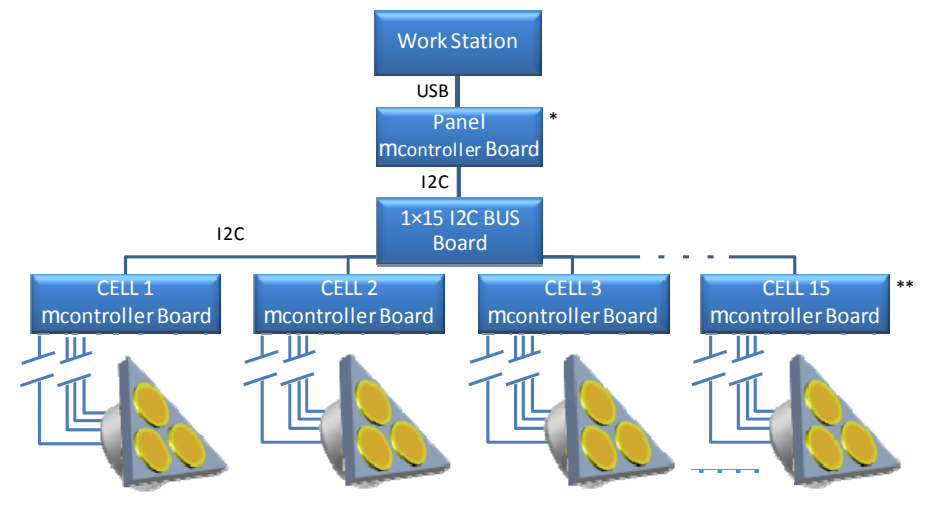

Fig 5. GEODA: Hardware architecture of the Control System.

The control software in Fig 6 is able to manage one panel for its measurement, characterization and calibration. Thus, the software has four tasks: satellite tracking, anechoic chamber measurements, S21 measurement of the RF circuit and the S21 measurement with the test signal and calibration of RF circuits.

Regarding the RF circuit scheme of the Cell in Fig 3, the bits of control are addressed through the update and control words in Fig 7. The update word uploads the state of the LNA and Phase Shifter of each patch of the 45 elements of the antenna as a parallel task during operation. 


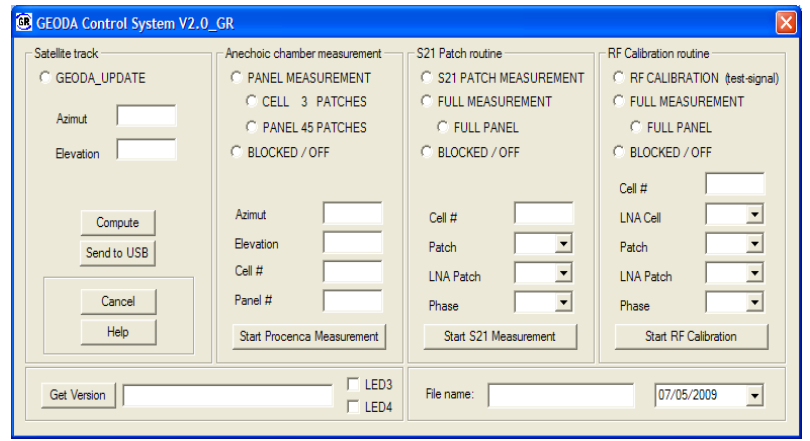

Fig 6. GEODA: Control Software interface.

The frequency of the update word depends on the minimum update time of the beamforming requirements for LEO satellite tracking and the subsequently tolerances of the gain error of the system. The control word uploads the state of the LNA and Switch of each Call of the triangular array.

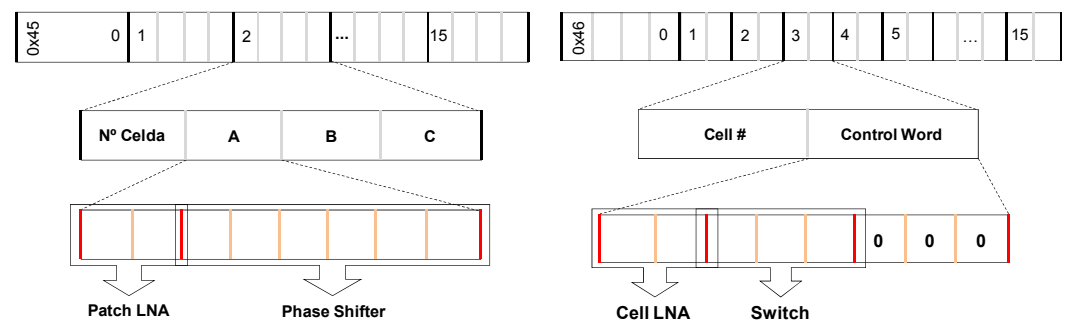

Fig 7. GEODA: Update (left) and Control (right) words.

\section{Conclusions}

A new conformal antenna with adaptive beam, working at $1.7 \mathrm{GHz}$ with double circular polarization is being developed. Radiating elements, T/R modules and control system of the whole antenna has been presented in this document. Next step will be measuring new T/R module and integrated it to a whole triangular array, checking performance of the system.

\section{Acknowledgments}

The authors wish to thank Ingeniería y Servicios Aeroespaciales (INSA) and Comisión Interministerial de Ciencia y Tecnología (CICYT) for supporting under the CROCANTE Project with reference TEC2008-06736-C03-01.

\section{References}

[1] M. Sierra Pérez, A. Torre, J. L. Masa Campos, D. Ktorza, and I. Montesinos, "GEODA: Adaptive Antenna Array for Metop Satellite Signal Reception," in Proc. 4th ESA International Workshop on Tracking, Telemetry and Command System for Space Application, Darmstadt, September 2007, pp. 1-4.

[2] M. A. Salas Natera, R. Martínez Rodríguez-Osorio, A. Antón Sánchez, I. García-Rojo, and L. Cuellar, "A3TB: Adaptive Antenna Array test-bed for tracking LEO satellites based on software-defined radio," in Proc. 59th International Astronautical Congress, Glasgow, October 2008, pp. 313-317.

[3] I. Montesinos-Ortego, J. L. Masa, M. Sierra Pérez, and J. L. Fernández-Jambrina. "Pyramidal adaptive antenna of planar arrays for satellite communications," EuCAP Proceedings, November 2007, Edinburgh, UK.

[4] I. Montesinos, M. Sierra Pérez, J. L. Fernández, R. Martínez and J. L. Masa. "GEODA: Adaptive Antenna of Multiple Planar Arrays for Satellite communications," EuCAP Proceedings, November 2009, Berlin, Germany.

[5] Marta Arias Campo, Ignacio Montesinos Ortego, José Luis Fernández Jambrina, Manuel Sierra Pérez. "T/R module design for GEODA antenna," EuCAP 2010, Barcelona, Spain.

[6] Miguel Salas, Ramón Martínez and Leandro de Haro. "Automated Measurement Procedure for the Calibration of Planar Active Arrays.” EuCAP 2010, Barcelona, Spain. 